\title{
Automatic Tuning of Spatially Varying Transfer Functions for Blood Vessel Visualization
}

Gunnar Läthén, Stefan Lindholm, Reiner Lenz, Anders Persson and Magnus Borga

\section{Linköping University Post Print}

N.B.: When citing this work, cite the original article.

C2012 IEEE. Personal use of this material is permitted. However, permission to reprint/republish this material for advertising or promotional purposes or for creating new collective works for resale or redistribution to servers or lists, or to reuse any copyrighted component of this work in other works must be obtained from the IEEE.

Gunnar Läthén, Stefan Lindholm, Reiner Lenz and Magnus Borga, Automatic Tuning of Spatially Varying Transfer Functions for Blood Vessel Visualization, 2012, IEEE Transactions on Visualization and Computer Graphics, (18), 12, 2345-2354.

http://dx.doi.org/10.1109/TVCG.2012.203

Postprint available at: Linköping University Electronic Press http://urn.kb.se/resolve?urn=urn:nbn:se:liu:diva-79365 


\title{
Automatic Tuning of Spatially Varying Transfer Functions for Blood Vessel Visualization
}

\author{
Gunnar Läthén, Stefan Lindholm, Student Member, IEEE, Reiner Lenz, \\ Anders Persson, and Magnus Borga, Member, IEEE
}

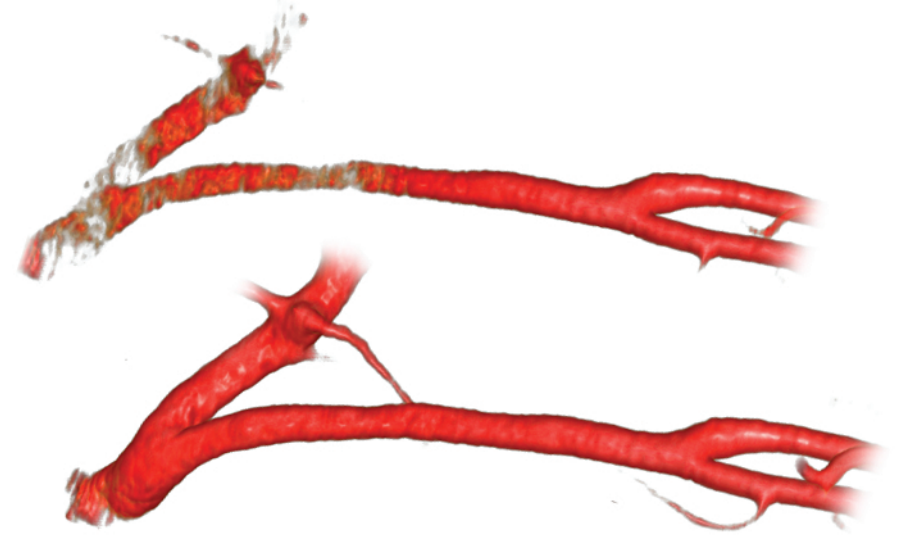

Fig. 1. Automatic adjustment of the Transfer Function for visualization of the carotid vessels in CT Angiography. Given a TF preset, the presented method locally shifts the preset for optimal visual vessel response. The intensity of the vessel in this example varies from left to right due to shifting contrast agent concentration. Because of this, the TF preset in the upper image fails to visualize the complete vessel structure. Our method compensates the intensity variation by locally shifting the preset, resulting in the lower image.

\begin{abstract}
Computed Tomography Angiography (CTA) is commonly used in clinical routine for diagnosing vascular diseases. The procedure involves the injection of a contrast agent into the blood stream to increase the contrast between the blood vessels and the surrounding tissue in the image data. CTA is often visualized with Direct Volume Rendering (DVR) where the enhanced image contrast is important for the construction of Transfer Functions (TFs). For increased efficiency, clinical routine heavily relies on preset TFs to simplify the creation of such visualizations for a physician. In practice, however, TF presets often do not yield optimal images due to variations in mixture concentration of contrast agent in the blood stream. In this paper we propose an automatic, optimizationbased method that shifts TF presets to account for general deviations and local variations of the intensity of contrast enhanced blood vessels. Some of the advantages of this method are the following. It computationally automates large parts of a process that is currently performed manually. It performs the TF shift locally and can thus optimize larger portions of the image than is possible with manual interaction. The method is based on a well known vesselness descriptor in the definition of the optimization criterion. The performance of the method is illustrated by clinically relevant CT angiography datasets displaying both improved structural overviews of vessel trees and improved adaption to local variations of contrast concentration.
\end{abstract}

Index Terms—Direct volume rendering, transfer functions, vessel visualization.

\section{INTRODUCTION}

CT Angiography (CTA) is a commonly used technique when diagnosing vascular diseases. To increase the contrast of the blood vessels, a contrast agent is injected into the patient prior to the scan. Ideally, the vessels can then be identified in the image data by values of around 300 Hounsfield units (HU). This identification becomes problematic as the

- All authors are with the Center for Medical Image Science and Visualization (CMIV), Linköping University, Sweden.

- G. Läthén, S. Lindholm, and R. Lenz are also with the Department of Science and Technology, Linköping University, Sweden, E-mail: \{gunnar.lathen,stefan.lindholm,reiner.lenz\}@liu.se.

- A. Persson is also with the Department of Medical and Health Sciences, Linköping University, Sweden, E-mail: anders.s.persson@liu.se.

- M. Borga is also with the Department of Biomedical Engineering, Linköping University, Sweden, E-mail: magnus.borga@liu.se.

Manuscript received 31 March 2012; accepted 1 August 2012; posted online 14 October 2012; mailed on 5 October 2012.

For information on obtaining reprints of this article, please send

e-mail to:tvcg@computer.org. concentration of the contrast agent often varies significantly throughout the vessels, causing the intensity values for blood to deviate from the ideal.

To understand the often complex geometry of the vascular tree, Direct Volume Rendering (DVR) techniques are commonly used to visualize the CTA data. For this, Transfer Functions (TFs) are used to map intensities in the CT data to color and opacity. With efficiency being a dominant problem in the clinical workflow [1, 20,26], clinical tools commonly provide predefined TFs (presets) for CTA. Unfortunately, the varying concentrations of contrast agent often causes these presets to yield less useful visualizations.

The goal of this work is to computationally automate, and improve, parts of the adjustment process for TF presets that are currently performed manually in CTA. Our concept starts with the traditional TF preset, adds additional components that analyze local vesselness and intensity variations and results in an improved TF model for CTA. Our approach is to mimic the manual procedure performed by a physician where the critical assessment of the TF preset is typically carried out by sliding the TF along the intensity value axis while visually observing what happens in the rendered image. In our implementation, the 
problem is formulated as an optimization which shifts the TF with the objective to maximize the perceived vesselness in the image. A key difference from similar works is that the vesselness computed for the optimization is performed after applying the TF (for a technical overview of the pipeline, see Section 3).

The main contribution of this work is an automated optimization method for CTA TF presets. The method increases both the usefulness and image quality of TF presets by reducing the necessary interaction and allowing the TF to be adjusted locally. The novelty in this work lies in the approach of performing a mixture of single and multi-scale vesselness analysis on both original and TF mapped data.

\section{Related Work}

There are two research areas related to our work. The primary area is TF design in direct volume rendering. The second area is blood vessel analysis and segmentation in general, where many sophisticated methods exist, see e.g. $[2,12,16]$ for review material. Using such methods for segmenting the blood vessels can be of great aid for diagnosis and decision support. Our work, on the other hand, targets the practical clinical workflow which is largely controlled by time constraints and the physician's required knowledge of technical algorithms. A major challenge is to achieve clinical acceptance which requires fast interaction, robustness and intuitive user parameters.

\subsection{Transfer Function Design}

The DVR technique [4] is a very common tool in the analysis of volumetric data. A key issue, as discussed in [24], is the design of TFs, which can be characterized as either data-centric or image-centric. The data-centric approaches derive information from the original data, and use this information to define TF parameters or guide the TF construction. The first ideas in [4] proposed a one-dimensional TF, based only on the intensity values of the data. Common extensions of this model add gradient magnitude $[10,17]$ or curvature $[8,11]$ as parameters to the TF. Other approaches use statistical information from local neighborhoods in the data $[6,15,23,31]$ to facilitate a more robust distinction between features of overlapping intensities. The above mentioned methods all rely on local neighborhood analysis, but objects can also be described on a higher abstraction level by e.g. shape, size and connectivity. This has been the focus of $[30,32,34]$ which use topological analysis of the data, and $[3,25]$ which use the estimated size and shape of objects. However, a general problem with multi-dimensional TF designs is the drastic increase in complexity for the user interaction, which requires good support in terms of interface widgets. This has been investigated in e.g. [13] and [28]. The work presented here relates to the above mentioned work in that the local neighborhood around a sample is used to enhance classification. There are two key points that separate our work from the other approaches: The first is that our pipeline contains components that analyze the data after the TF has been mapped rather than the raw data directly. The second is that we do not expose the value from the computation as a free dimension when constructing the TF.

For the visualization of medical data, it is advantageous to use domain knowledge in the TF design. Semantically separating different $\mathrm{TF}$ primitives (or different TFs) depending on the tissues they are meant to visualize allows additional information to be encoded in the TF. One example is spatial relations between tissue types $[18,19,21,27]$. The work presented here shares a similar approach in that parts of the TF that dictate the visual appearance of vessels are treated differently than those for other tissues.

Image-centric approaches to TF design use information in the rendered image for controlling the TF. The pioneering work in this category was [7] which generates a TF using a stochastic optimization algorithm. The optimization is either controlled by the user by selecting a preferable set of rendered images, or by an objective function. A similar user interface idea was the "gallery" approach in [22]. More recent work includes [33] which allows editing and combining images rendered by different TFs. Our approach is similar to image-centric methods, in the sense that we analyze the TF mapped intensity values to guide the TF design, rather than the original data. A key difference, however, is that our analysis is performed in the 3D object space rather than in the rendered image. Compared to [7], our method greatly reduces the number of free parameters in the TF optimization, by using domain knowledge of the application. We also perform a local optimization which results in a spatially varying field of locally optimized TFs.

Turning to the particular application of blood vessel visualization, the recent work by Kubisch et al. [14] provides a good overview of the problems and reviews related work. Particularly related to our work is the idea by Joshi et al. [9] which also employs a vesselness measure to improve visualization. They propose a novel vesselness measure and investigate the clinical effectiveness of different lighting models. The vesselness is used to "boost" the opacity of low intensity structures which typically are below the threshold of the TF. The key difference with our work is that we use the vesselness measure as a guide to locally shift the TF to these lower intensities. Therefore, we do not depend directly on the vesselness in the visualization and are not strongly sensitive to typical issues with weak vesselness response at branching points in the vascular tree. We will compare our resulting visualization with the ideas presented by Joshi et al. [9] in Section 7.

\subsection{Vesselness Measures}

As will be detailed in subsequent sections, we use a vesselness measure in the formulation of the problem to determine an object's similarity to a vessel structure. Much recent work has focused on multi-scale methods based on eigenvalue analysis of the Hessian [16]. Following this common approach, we have implemented the most recently proposed measure by Sato et al. [29]. For completeness we include the expression for the vesselness $V$ in Appendix A.

Since vessels typically are varying in size, it is common to measure the vesselness on different scales. The smallest scale should be tuned to detect the smallest vessel structures in the data with as little influence from noise as possible. The largest scale should be tuned to the largest vessel. Sato et al. [29] use a maximum operation to compute the multi-scale vesselness response:

$$
V=\max \left\{V_{\sigma_{i}}: \sigma_{i} \in \Sigma\right\}
$$

where $V_{\sigma_{i}}$ is the vesselness for a single scale and the range of scales $\Sigma$ is typically chosen as $\left\{1, \sqrt{2}, \ldots,(\sqrt{2})^{n}\right\}$ in which $n$ represents the coarsest scale at which all the vessels are detected.

\section{Pipeline OVerview}

With the concept to mimic the manual process of TF interaction, our approach is based on measuring the visual impact for varying TF settings. In the context of this work, we define the term "visual response" as the output volume of the TF mapping, disregarding any viewing and shading parameters in the remaining rendering pipeline.

We first introduce a TF model that allows us to shift the TF along the intensity axis. An optimal TF setting is then found by varying the shift while measuring the visual response in an iterative optimization process repeating the following steps (red arrows in Figure 2).

\section{Update the TF shift}

2. Apply the (shifted) TF to the data

3. Measure the perceived vesselness in the data for the current TF

In step 3, a single-scale vesselness filter is used to characterize the structure and size of the objects that become visible from the TF mapping. The process favors TF parameters that maximize the size of vessels under the constraint that they maintain their vesselness (as defined by the filter).

After the optimization, a multi-scale analysis of the original data is performed to determine the amount of vesselness in a region independently of vessel size. This is used to localize and regularize the parameter field. An overview of the pipeline is illustrated in Figure 2. 


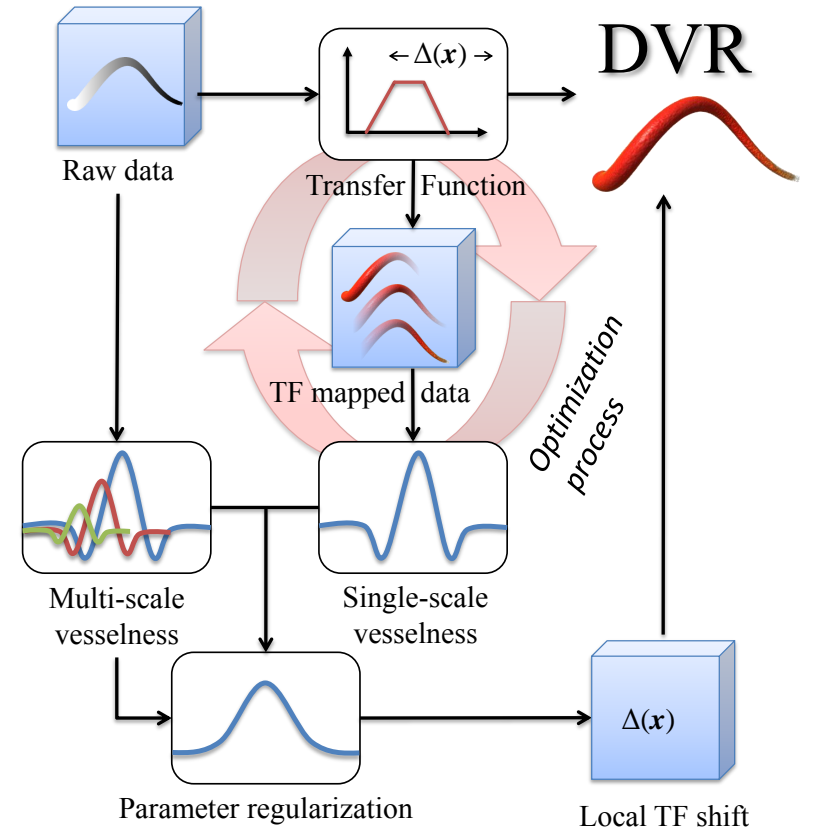

Fig. 2. Pipeline overview. Local shifts for the Transfer Function are automatically tuned through an optimization process based on single-scale vesselness analysis of the TF mapped data. The local shifts are then localized and regularized using multi-scale vesselness analysis of the original data. The final result is a single scalar value per voxel, describing the TF shift that maximizes the visual representation of vessels.

\section{Locally Optimized Transfer functions}

In CTA, the goal of the physician's TF interaction is to find the TF setting which shows the complete extent of a vessel and facilitates accurate estimation of vessel geometry. For a given region of interest, this problem of intensity variations in the blood can be solved visually by the physician by sliding the TF towards lower intensities until the visual response (the thickness) of the vessel no longer increases or the background emerges to obscure the vessel.

To illustrate this phenomenon, we will use a volume of synthetic vessels of varying intensity, with the middle slice of the data shown in Figure 3. Due to the varying intensity for the upper vessel, the TF preset applied in Figure 3(b) is unable to visualize the complete structure. In Figure 3(c) we see the best result obtained for the low intensity parts of the vessel by sliding the TF downwards to lower intensities. However, for this setting, the non-vessel object in the left part of the volume appears and occludes the vessels.

Our approach aims to mimic the TF design process performed manually by a physician. For this purpose we modify the standard 1D (intensity) TF model:

$$
T F(\boldsymbol{x}, I(\boldsymbol{x}), \Delta(\boldsymbol{x}))=T F_{\text {preset }}(I(\boldsymbol{x})-\Delta(\boldsymbol{x}))
$$

Here, $\boldsymbol{x}$ is the spatial coordinate, $I(\boldsymbol{x})$ is the intensity and $\Delta(\boldsymbol{x})$ is the free parameter of the TF. Thus, the TF is represented by the initial condition $T F_{\text {preset }}$ with an argument shifted by $\Delta(\boldsymbol{x})$. Note that the shift $\Delta$ depends on the spatial position and therefore, unlike in the case of manual interaction, allows the TF to vary spatially. To simplify notation, we will use $T F\{\Delta(\boldsymbol{x})\}=T F(\boldsymbol{x}, I(\boldsymbol{x}), \Delta(\boldsymbol{x}))$. For CTA data, $\Delta(\boldsymbol{x})$ has a physical interpretation in terms of Hounsfield units (HU).

\subsection{The Objective Function}

An important aspect when computing the vesselness measure is the scale. In fact, the vesselness is estimated by a set of Gaussian derivative filters which, by design, result in higher magnitudes in the filter response for a particular vessel size. In cases where it is advantageous to have a vesselness measurement that is independent of vessel size,

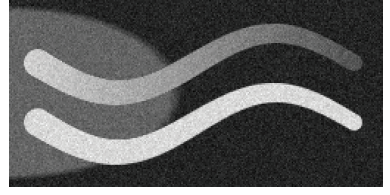

(a) Slice view

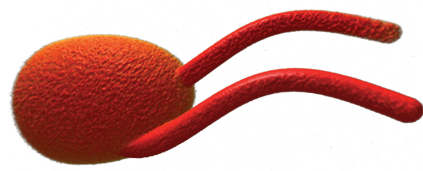

(c) Globally shifted TF preset

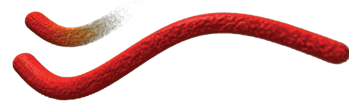

(b) $\mathrm{TF}$ preset

(d) Locally shifted TF preset

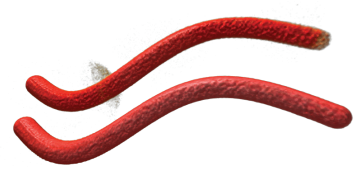

Fig. 3. Synthetic vessels of varying intensity, with the middle data slice shown in (a). The rendering in (b) shows a preset TF which fails to capture the lower intensities of the upper vessel. Globally shifting the preset in (c) reveals the upper vessel, but clutters the view with the nonvessel object. By applying our pipeline, we can tune spatially varying shifts of the TF to visualize the complete vessels as shown in (d).

the responses of several (typically hierarchically sized) filters are often compared in what is referred to a multi-scale approach. In contrast to this, the optimization step in this work is inherently size-dependent with the aim to maximize the size of a vessel's visual response by shifting the TF. For this purpose we use a single-scale approach. By only using a single scale, it is possible to relate the magnitude in the filter response to the size of the visual representation for a vessel. This effectively favors the higher magnitude responses of larger vessel representations (thicker versions of the same vessel). A necessary requirement for this is that the chosen filter corresponds to a larger vessel size than what can be achieved through the optimization (the size of the largest visual representation of any vessel). This motivates the formulation of the problem as a maximization of vesselness in the TF mapped data by:

$$
\underset{\Delta(\boldsymbol{x})}{\arg \max } V_{\sigma}\{T F\{\Delta(\boldsymbol{x})\}\} \text { subject to }\left\{\begin{array}{l}
T F_{\text {low }}-\Delta(\boldsymbol{x}) \geq I_{\min } \\
T F_{\text {high }}-\Delta(\boldsymbol{x}) \leq I_{\max }
\end{array}\right.
$$

where $T F_{\text {low }}$ and $T F_{\text {high }}$ are the lower and upper intensity ranges for the TF preset. Thus, the shifted TF preset is restricted to lie between the intensities $I_{\min }$ and $I_{\max }$. For CTA data, these parameters can intuitively be interpreted in terms of Hounsfield units and can be kept at fixed values.

To search for the optimal parameters $\Delta(\boldsymbol{x})$, any suitable optimization method can be used. For our initial work so far, we use a simple exhaustive search of the interval $\left[I_{\min }, I_{\max }\right]$ by sampling the objective function at a given number of equally spaced positions. Using the synthetic example in Figure 3, the resulting parameters from the optimization is shown in Figure 4(a).

\subsection{Localization and Regularization}

The optimized value is only significant in regions where there are vessel-like structures appearing from the TF mapping. For regions with non-vessel structures, the vesselness filter will still give a nonzero output, albeit with very small magnitude. If there are no other dominant vessel structures in the region, these small magnitude responses will still be considered optima and visualized by the corresponding TF mapping. To localize the TF shifts to regions that include only significant vessel structures, the parameter field is weighted by a vesselness measure at each point. This vesselness is computed through a multi-scale analysis of the unmapped data, $V(I(\boldsymbol{x}))$ as in Eq. (1). Utilizing a multi-scale approach in this step ensures that the computed vesselness measure, unlike in the optimization, is size-independent. The weighting of the parameters is performed by:

$$
\Delta_{m}(\boldsymbol{x})=\Delta(\boldsymbol{x}) m(\widetilde{V}(I(\boldsymbol{x})))
$$



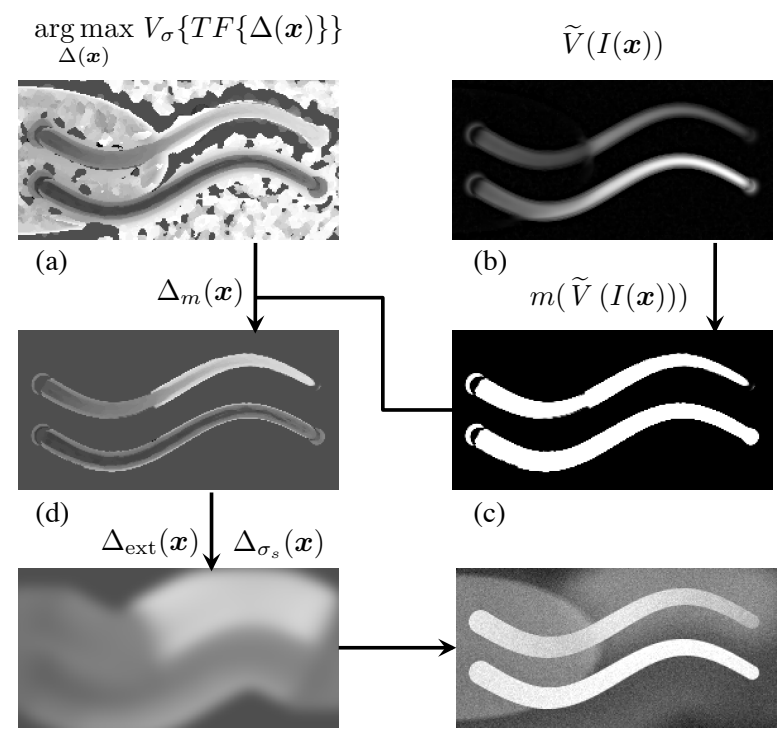

(e)

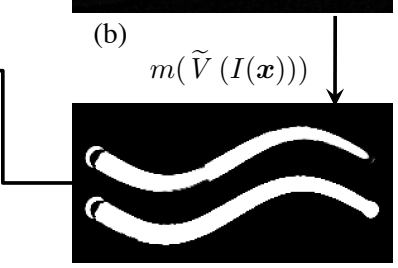

(c)

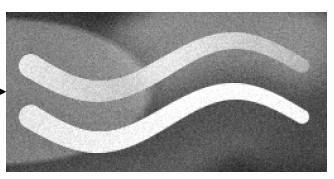

(f)

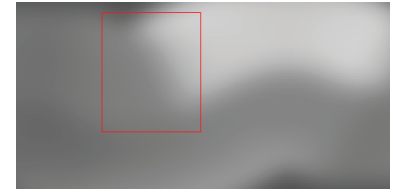

(a) Regularization using $\sigma_{s}=8$

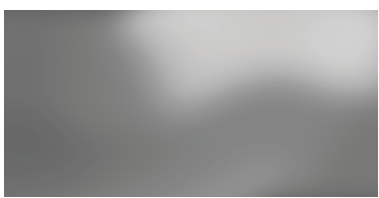

(c) Regularization using $\sigma_{s}=10$

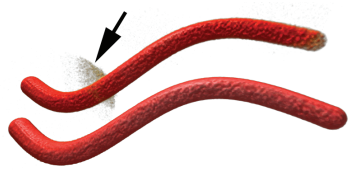

(b) TF mapping $\sigma_{s}=8$

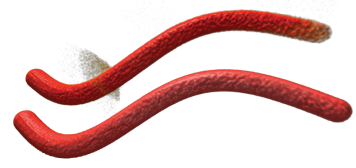

(d) TF mapping $\sigma_{s}=10$

Fig. 5. Illustrates the behavior for different sizes of regularization kernels. Regularization using a kernel of $\sigma_{s}=5$ is shown separately in Figure 4(e) resulting in the rendering in Figure 3(d). Increasing the regularization as in (a) and (c) results in more slowly varying TF parameters. However, larger regularization kernels average parameters over larger regions and transition effects, such as the ring pointed out in (b) can become more apparent.

Fig. 4. Overview of each step of the method. First, the optimization finds the parameters which, at each point, maximize the vesselness for the TF mapped data (a). Then, the vesselness of the original data is computed and used to maintain only the parameters of significant vesselness (b,c,d). Finally, the parameters are regularized (e) and can be used to either shift the TF or the original data (f).

where $\widetilde{V}(I(\boldsymbol{x}))$ is the normalized vesselness measure, and $m \in[0,1]$ is a sigmoid mapping function given by:

$$
m(\widetilde{V}(I(\boldsymbol{x})))=\frac{1}{1+e^{-(\widetilde{V}-b) a}}
$$

In this work we use typical values $b=0.04$ and $a=200$ which gives a soft thresholding operation. This effectively suppresses optimized parameters for low-vesselness structures, while maintaining the parameters for vessels. The results of the vesselness computation and mapping are shown in Figure 4(b,c). Then, weighting the optimized parameters using Eq. (4) gives the result in Figure 4(d).

Neither the objective function nor the optimization include any method for regularizing the parameters. This means that there is nothing, other than voxel size, that limits how fast the TF is allowed to change spatially. If the TF is allowed to change rapidly, there is an apparent risk that sudden changes create unreliable visual results, and artificially created edges. This risk is effectively reduced by explicitly regularizing (smoothing) the parameters. We perform this in a twostep process. The first step consists in extrapolating (extending) the optimized parameters into non-vesselness regions. By doing this, vessels which are small are effectively allowed to control the parameter setting of a larger neighborhood. The second step imposes the actual regularization by a low-pass filtering.

Starting with the extension operation, it is implemented by a weighted filtering of the parameter $\Delta_{m}$ :

$$
\Delta_{\mathrm{ext}}(\boldsymbol{x})=\frac{\int_{\boldsymbol{y} \in \Omega_{I}} \Delta_{m}(\boldsymbol{y}) m(\boldsymbol{y}) G_{\sigma_{s}}(\boldsymbol{x}-\boldsymbol{y}) d \boldsymbol{y}}{\int_{\boldsymbol{y} \in \Omega_{I}} m(\boldsymbol{y}) G_{\sigma_{s}}(\boldsymbol{x}-\boldsymbol{y}) d \boldsymbol{y}}
$$

where $\Omega_{I}$ is the domain of the image. Here, the mapping function $m$ (Eq. (5)) is used as weight (or certainty) and the extension kernel $G_{\sigma_{s}}$ is a Gaussian function of standard deviation $\sigma_{s}$. Intuitively, this operation can be interpreted as a dilation of the parameter field. By using a Gaussian extension kernel, we achieve a low-pass filtering in regions with non-zero certainty. However, in transitions to zero certainty regions, the filtered output will rapidly decrease to zero. Therefore, the second step applies low-pass filtering using standard convolution with

a Gaussian kernel to get the final regularized parameter field:

$$
\Delta_{\sigma_{s}}(\boldsymbol{x})=\int_{\boldsymbol{y} \in \Omega_{I}} \Delta_{\mathrm{ext}}(\boldsymbol{y}) G_{\sigma_{s}}(\boldsymbol{x}-\boldsymbol{y}) d \boldsymbol{y}
$$

The extension in Eq. (6) and the regularization in Eq. (7) can use different kernels. However, it should be noted that the size of the extension kernel in Eq. (6) must be at least as large as the regularization kernel in order to preserve the optimized parameters. If the extension kernel is smaller than the regularization kernel, the optimized parameter will be averaged over both suppressed and non-suppressed parameters, which gives a bias in the parameter towards zero. For example, consider the parameters maintained in Figure 4(d) for the low-intensity vessel in the upper right part. If the parameters are not extended prior to regularization, the optimized parameters (bright) will be averaged with the suppressed parameters (dark). By first extending the parameters, we preserve the optimized parameter values in the regularization shown in Figure 4(e). Note that the gray-values in Figure 4(d) and Figure 4(e) are given in the same scale and can be directly compared.

As an example, we assume that we have only one TF primitive which is shifted according to the optimized parameters. We observe that applying a spatially varying shift of this TF is equivalent to shifting the original data and applying the unshifted TF globally. In fact, the rendering in Figure 3(d) is produced by this idea of shifting the data. In Figure 4(f) we see a slice of this data, which in practice is the sum of Figure 3(a) and Figure 4(e). By this, our method can be interpreted as an intensity equalization of vessel structures. Generally however, we need to maintain the original data for additional TF primitives which are not subject to local shifts, e.g. for simultaneously visualizing other types of tissues.

For the regularization in Figure 4(e) we used a Gaussian kernel of standard deviation $\sigma_{s}=5$ voxels. To compare the result using different regularization kernels, we show the regularized parameters and the resulting TF mappings also for $\sigma_{s}=\{8,10\}$ voxels in Figure 5 . Note that the TF mapping using $\sigma_{s}=5$ is given in Figure 3(d). For all parameter images, we can see that the upper vessel object requires a shift of the TF to lower intensities (indicated by bright gray values in Figure 4(e) and Figure 5(a,c)). Relating to the non-vessel object visible in Figure 3(c) we can also see that the shift parameter varies more abruptly as the upper vessel structure leaves this object (the region marked with a box in Figure 5(a)). This indicates that the shift parameter which gives the most complete extent of the vessel is selected in the optimization. In other words, in the region outside of the non-vessel object we can shift the TF further to lower intensities without the background obscuring the vessel. A visual consequence of this transition in the shift parameter is the "ring" pointed out by the arrow 
in Figure 5(b) which shows the tip of the non-vessel object. We have not observed this effect in any of the real datasets, but we note that this transition ring becomes more apparent for larger regularization kernels when comparing Figure 3(d) and Figure 5(b,d).

\section{IMPLEMENTATION DETAILS}

Once the optimal parameter is computed for each voxel it is generally stored in a separate floating-point volume of the same dimensions as the data. Using the parameter during rendering in this case amounts to an additional texture lookup per voxel prior to the TF mapping. Only the TF primitive used for visualizing the blood vessels is shifted by the optimized parameters, while other TF primitives used for e.g. bone or fat visualization are stationary. The vesselness was estimated using the original approach in [29], i.e. by filtering the volume with differently sized Gaussian derivative kernels. This is done efficiently using separable convolutions on the GPU.

An implementation of the method was made in Matlab with CUDAsupported computations of the vesselness and the regularization. Voreen was used as a front-end to visualize the result. Example code can be found online at http://dmforge.itn.liu.se/vis2012.

\section{EXPERIMENTS}

To illustrate the behavior of our method, we have used one phantom CT volume and two CT angiography datasets. This section will start by describing the parameter setup, the datasets and the main visualization problem, and then continue with the results. For the two CTA datasets, we have added a ramp TF for bone and a tent TF for skin for context in the visualization. Note that these are not affected by the optimization but remain fixed along the intensity axis.

Parameters. For all experiments, we use a trapezoidal TF preset with a width of 200 Hounsfield units (HU). In the optimization (Eq. (3)) the TF is allowed to shift in the interval $\left[I_{\min }=0, I_{\max }=500\right]$ which is a typical range for contrast injected blood vessels. We sampled the objective function (Eq. (3)) uniformly at 30 positions along this interval. The regularization in Eq. (7) uses a kernel of standard deviation $\sigma_{s}=8$ for all examples. The scale at which the vesselness is computed is adapted to each case, and given below.

Example 1: Phantom. The phantom volume is a CT scan of a liquid filled bucket with plastic tubes. The Hounsfield values of the liquid and the plastic tubes correspond to living soft tissue and blood vessel walls, and the plastic tubes have been injected with a contrast agent. However, the contrast agent concentration, and therefore the Hounsfield values, varies in the tubes which gives only partial visualizations of the complete structures when applying the TF preset, centered around $300 \mathrm{HU}$, as shown in Figure 6(a). The vesselness filter use kernels with $\sigma=4$.

Example 2: Carotid CTA. The first CTA dataset is an examination of the carotid vessels. In this examination, the purpose is to inspect the carotid vessels, marked with black frames in Figure 8(a) and Figure 8(b). Visual inspection of the data slices shows that the Hounsfield values for the vessels are increasing from the aorta up towards the brain. Therefore, the TF preset centered around the upper intensity values $(450 \mathrm{HU})$ fails to visualize the complete vessels in the lower black frame in Figure 8(a). The vesselness filter use kernels with $\sigma=4$.

Example 3: Leg CTA. The second clinical volume is a CTA of calcified vessels in the leg, shown in Figure 9. Due to the calcification, many vessels have low intensity and are missing from the TF preset visualization in Figure 9(a). This example uses a TF preset centered around $300 \mathrm{HU}$ and a vesselness filter with kernels of $\sigma=2$.

Comparison. To place our results in context with related work, we compare our renderings with the ideas presented by Joshi et al. [9]. In their work, the opacity of a voxel is "boosted" depending on its vesselness measure. The idea is to reveal structures of low intensity but high vesselness, which would typically be hidden by the TF. In our implementation of their idea, we use the weighted multi-scale vesselness mapped by Eq. (5) with parameters $b=0.06$ and $a=150$. This mapped vesselness is then added to the opacity of each voxel by a factor so that a visually informative rendering is obtained.

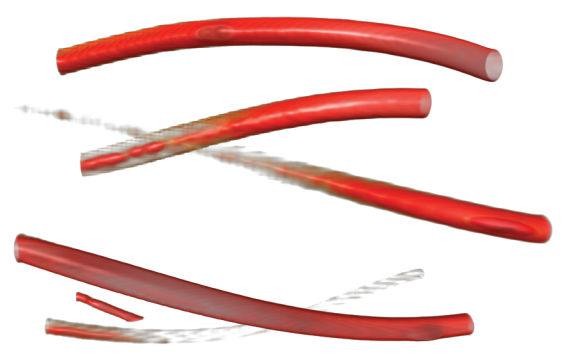

(a) $\mathrm{TF}$ preset

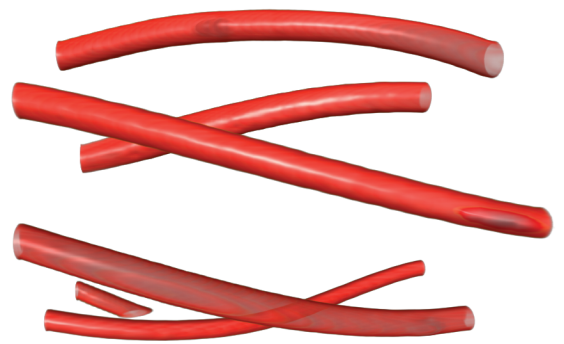

(b) Locally shifted TF preset

Fig. 6. This example shows a rendering of a physical phantom, in which the intensity varies in the tubular structures. The TF preset is unable to visualize the complete intensity range of the structures as shown in (a). The locally shifted TFs resulting from our method gives a more complete rendering of the structures as shown in (b).

\section{RESULTS AND DISCUSSION}

Volume renderings of the physical phantom are shown in Figure 6 for the TF preset and the locally shifted TF respectively. Furthermore, slices in Figure 7 show TF mapped data and the spatially varying parameters of the shifted TF. In Figure 6(a) we see that the TF preset does not represent the wide range of intensities in the data and fails to render the complete structures. This is also evident from the slice image of the TF preset mapping in Figure 7(a). The locally shifted rendering in Figure 6(b) reveals more complete structures, which shows that the method can correct for the spatially varying intensities of the tubular structures. Note that the regions visible in the parameter image in Figure 7(c) correlate to the different structures in Figure 7(d). It should also be remarked that the brightest structure in Figure 7(d) is outside the range $I_{\max }$ in the optimization, and therefore only the transition region between the interior of the tube and the background is captured in Figure 7(b).

For the carotid dataset, we can see in Figure 8(a) that the TF preset fails to visualize the complete carotid vessels as they descend towards the aorta. The lower parts of the vessels are however evident in the locally shifted TF mapping resulting from the optimization in Figure 8(b). In this example, the TF preset is centered around the upper intensity values and is not wide enough to cover the complete intensity range of the vessels. This can be compensated by widening the TF, with a risk of introducing additional objects in the visualization. Instead, our automatic method maintains the width of the TF, but shifts the TF locally to reveal as much as possible of the vessels. By this, we are less likely to clutter the view with objects not relevant for the visualization. This example illustrates the method's capability of correcting the preset TF for a real-world dataset of clinical importance.

For the leg CTA dataset, the TF preset Figure 9(a) fails to reveal many of the small vessels due to low contrast agent concentrations. Applying our optimization approach gives the result in Figure 9(b). We have indicated a slice in Figure 9(a) by a black frame for which the data and the TF shift parameters $\Delta_{\sigma_{s}}(\boldsymbol{x})$ are shown in Figure 9(e) and Figure 9(f). In these images we can correlate the vessel structures 

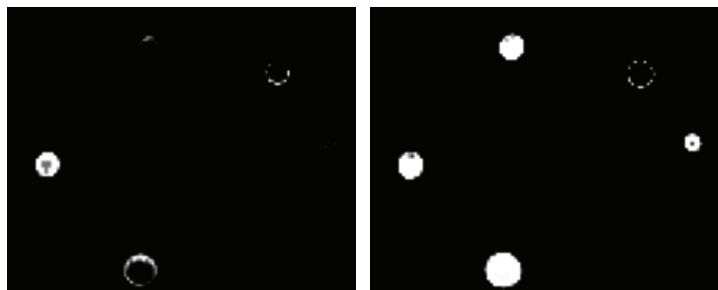

(a) $\mathrm{TF}$ preset

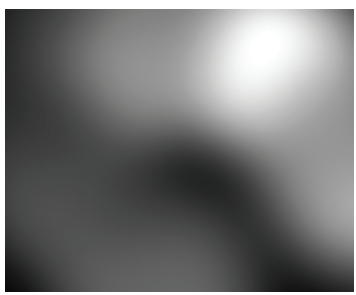

(c) TF parameters (shifts) (b) Locally shifted TF preset

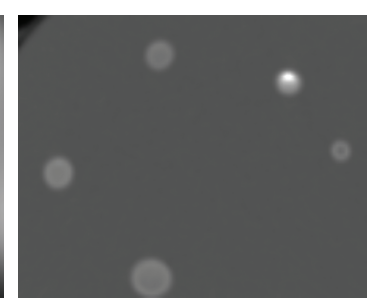

(d) Data

Fig. 7. Slice of the physical phantom, showing the preset and locally shifted mappings in (a) and (b). The TF parameters $\Delta_{\sigma_{\mathrm{s}}}(\boldsymbol{x})$ are given in (c), where bright and dark intensities indicate TF shifts towards $I_{\min }$ and $I_{\max }$ respectively. The different regions in the parameter image can be correlated to the corresponding features in the original data in (d). Note that the bright structure in (d) is above the range $I_{\max }$ so only the boundary of this tube is captured.

in the data by the affected regions in the parameters (for example the circled regions). We note that our localization correctly maintains the optimized parameters for the blood vessels and that the regularization produces a slowly varying field. In our experiments, we have generally noted that the vesselness filter typically gives a response for ring structures and other irregularities commonly seen at the surface of bones. This will be discussed further in Section 7.1. The optimized result in Figure 9(b) can be compared to globally shifting the TF preset to lower intensities until the muscle tissue appears, in Figure 9(c). By doing this we also achieve a more complete view of the smaller vessels, but at the expense of visual clutter from the muscle tissue. Even if Figure 9(c) shows more vessel structures than Figure 9(b) we emphasize that Figure 9(b) is produced by an automatic process, which can be preferred if the manual operation is seldom performed. That being said, it can still be beneficial to explore the optimized result in certain situations. To show that this is possible, Figure 9(d) shows a visualization where the optimized parameters are globally shifted and scaled. Here we can see most of the vessels also visible in Figure 9(c) but without the visual clutter. The shift-and-scale operation is very similar to a window/level-tool, commonly used for 2D medical images. This similarity gives us reason to believe that this can be an acceptable operation for the end user. We stress that this idea is preliminary and will be further explored in future user studies.

Figure 10 shows the results of another vessel visualization technique [9] also based on the vesselness measure. In contrast to our work, this method uses the vesselness directly in the visualization pipeline to modify the opacity of the rendered voxels. This approach makes a strong connection between the quality of the vesselness estimation and the produced rendering, and can introduce problems especially at branch points. It should be noted that in the original work [9], the authors implemented a different vesselness measure with better robustness to branch points, so our results based on the vesselness filter by Sato et al. [29] cannot be directly compared to the results in their paper. Comparing the renderings in Figure 10 with our results in Figure 8 and Figure 9, we see that both methods succeed in revealing the main missing vessel structures, albeit with a little more visual clutter in Figure 10. An important difference is that the renderings in Figure 10 are produced by manually exploring one parameter which steers the opacity based on vesselness, whereas our method is automatic. The most appropriate comparison is between Figure 10(b) and Figure 9(d)

Table 1. Timings for the datasets used in the experiments. The dataset sizes are extended to multiples of 32 in the actual computation on the GPU. The vesselness computation is repeated for each sampling of the objective function Eq. (3) (30 times for all experiments), while the regularization is performed only once. The total time gives the complete pipeline in Figure 2 for computing the TF shifts.

\begin{tabular}{r|c|c|c|c} 
Dataset & Dimensions & Vesselness & Regularization & Total \\
\hline Phantom & $90 \times 111 \times 72$ & $75 \mathrm{~ms}$ & $192 \mathrm{~ms}$ & $5.1 \mathrm{~s}$ \\
Carotid & $346 \times 213 \times 206$ & $392 \mathrm{~ms}$ & $452 \mathrm{~ms}$ & $19.7 \mathrm{~s}$ \\
Leg & $192 \times 379 \times 581$ & $610 \mathrm{~ms}$ & $1412 \mathrm{~ms}$ & $31.5 \mathrm{~s}$ \\
\hline
\end{tabular}

in which we have included manual control also for our method. These images show only small differences visually, but conceptually they are very different. In fact, Figure 10(b) is largely a visualization of the vesselness rather than the raw data which questions the usefulness when assessing e.g. vessel geometry for diagnostic purposes. For this, it is required that the user understands the relation between the raw data and the vesselness filter response. Our method indirectly uses the vesselness to compute a shift of the TF (or the data) which has a much simpler interpretation and is closely related to the original data.

Timings for the different datasets are listed in Table 1. In this initial work we performed all computations on the full datasets. We expect significant performance improvements for an optimized pipeline when computations are restricted to only significant vessel regions, determined by the vesselness mapping function in Eq. (5). For the real carotid and leg CTA datasets, only $5.8 \%$ and $1.1 \%$ respectively of the voxels are above the typical significance threshold of $b=0.04$ This, and other computational optimizations, are considered part of future work. It should also be noted that, since the visualization can be explored interactively after our computations, it is possible to precompute the TF parameters for a number of datasets in a batch and perform the diagnosis on the entire set efficiently at a later stage.

\subsection{Limitations of the Method}

This section outlines some of the limitations of the presented method. In all cases, the limitations have been discussed with clinical professionals who remain positive that the improvement in image quality are worth the potential trade-offs that might occur.

False emphasis on vessel-like features: The method's proficiency to distinguish vessels from other vessel-looking features is fully dependent on the vesselness filter. In short, the filter roughly characterizes vesselness as "having a cylindrical shape" and is known to sometimes misclassify other cylindrical features, such as bones and tendons. For a more complete discussion on the strengths and weaknesses of the vesselness filter see [5, 29]. What can be said is that, from the physicians point of view, a "falsely" emphasized structure is in this case itself typically not a problem as domain experts are very good at differentiating between anatomical structures. It does become a problem if the "falsely" emphasized feature obstructs the view of more important structures, although this is not considered a major issue. As mentioned for the leg dataset discussion in the previous section, bone structures tend also to conflict with nearby vessels of low intensity. Since the vesselness filter gives some response for the bone structure, it may prevent a TF shift necessary to completely visualize the vessel. In this case, the regularization determines the outcome. If the regularization kernel does not cover the vessel and bone simultaneously, the parameter for the vessel will be completely preserved and the vessel fully visualized. For larger kernels, the parameters of the bone and vessel will be combined, so that we will see some blend between the bone and vessel parameters. However, we can still argue that our method improves over the TF preset: If the vessel was already revealed by the TF preset, our method will not induce any shift and not change the result. If, on the other hand, the vessel was not visualized by the TF preset, manual correction is required anyhow. It is however likely that the correction needed is smaller when using our method. Future work will study this issue and investigate whether additions to the vesselness filter can reduce the response of bones. 


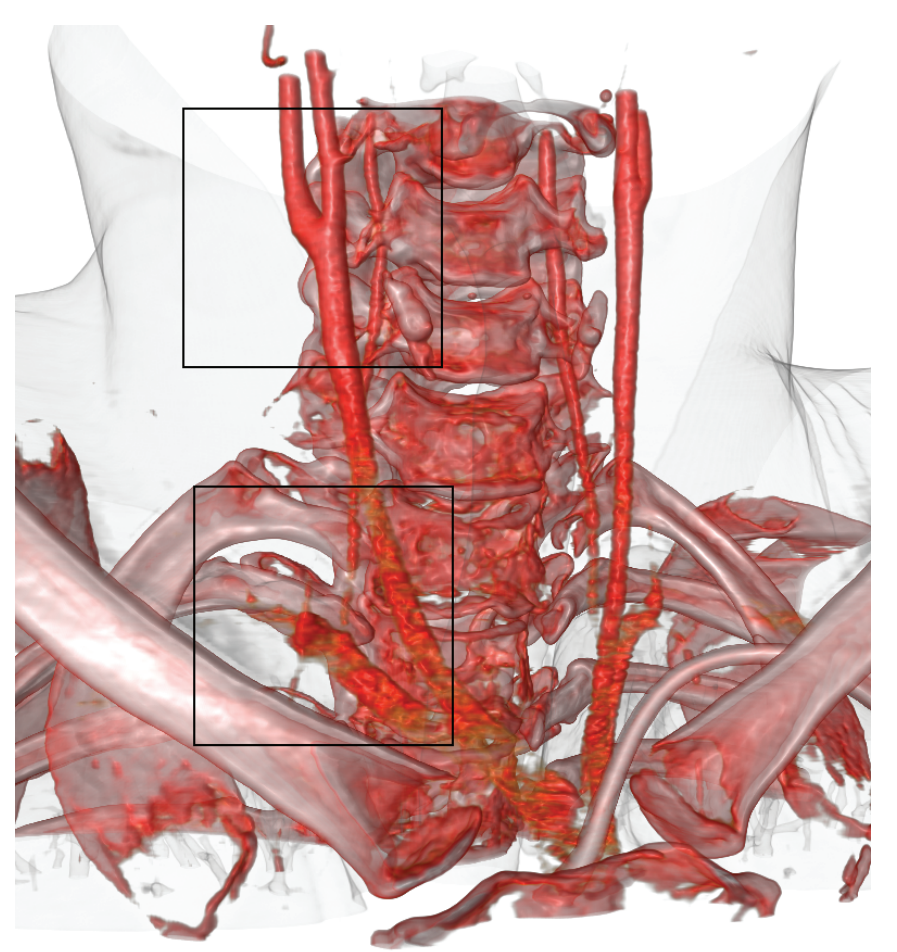

(a) Original TF preset
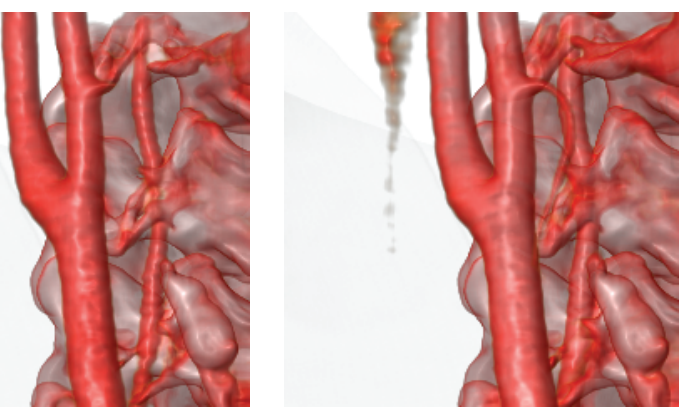

(c) Close-up comparisons of upper part of the artery in images $a$ (left) and $b$ (right). The original preset works well in this region and the optimized shift is therefore low. Note however the appearance of the structure to the upper left, which is a vein of low intensity.

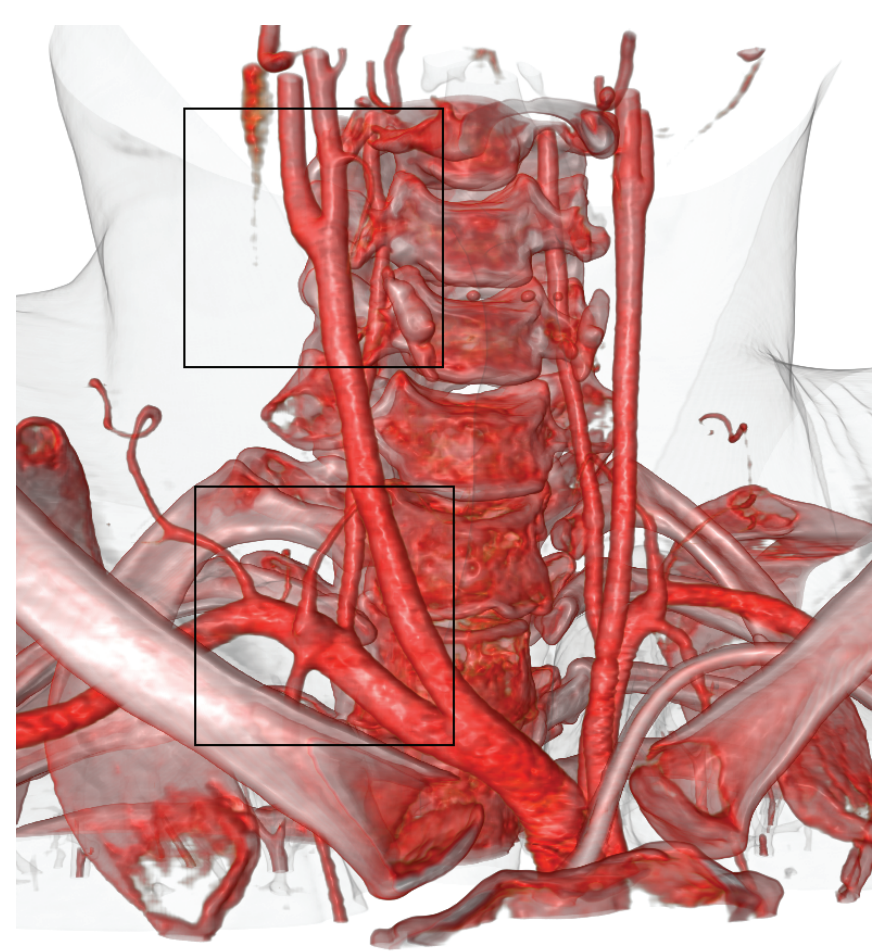

(b) Locally shifted TF preset
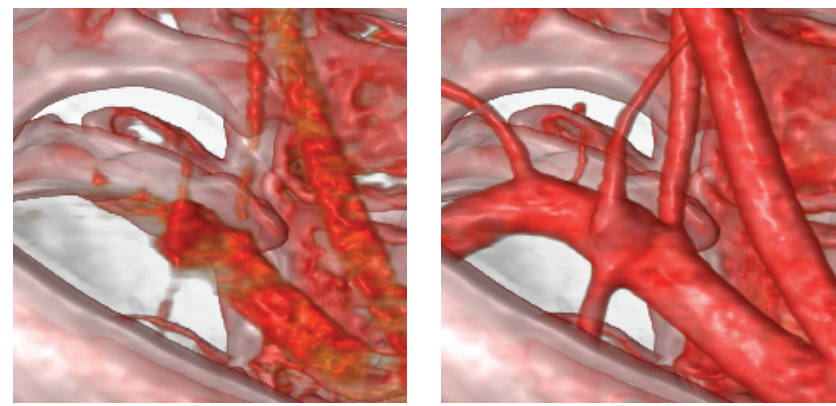

(d) Close-up comparisons of lower part of the artery in images $a$ (left) and $b$ (right). Thanks to local adjustments, the locally shifted TF maintains good results under varying intensity.

Fig. 8. CT Angiography visualization of the carotid arteries. Varying mixture concentrations between blood and contrast agent yields varying intensities in the data. This causes traditional TF presets to fail capturing the full extent and size of the targeted vessels. The method presented in this paper locally optimizes (shifts) the TF preset such that it is valid in more than a single region. (The black frames in $a$ and $b$ indicate the regions used for the close-ups in $c$ and $d$.) 


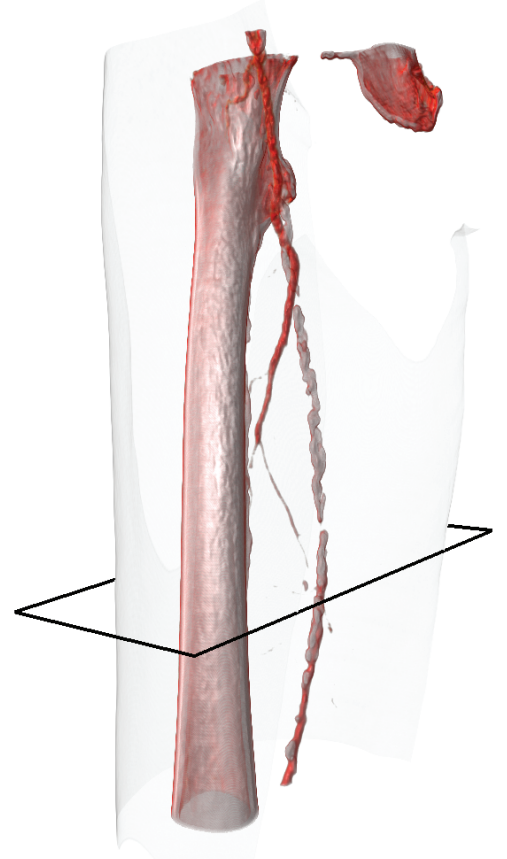

(a) Original TF preset

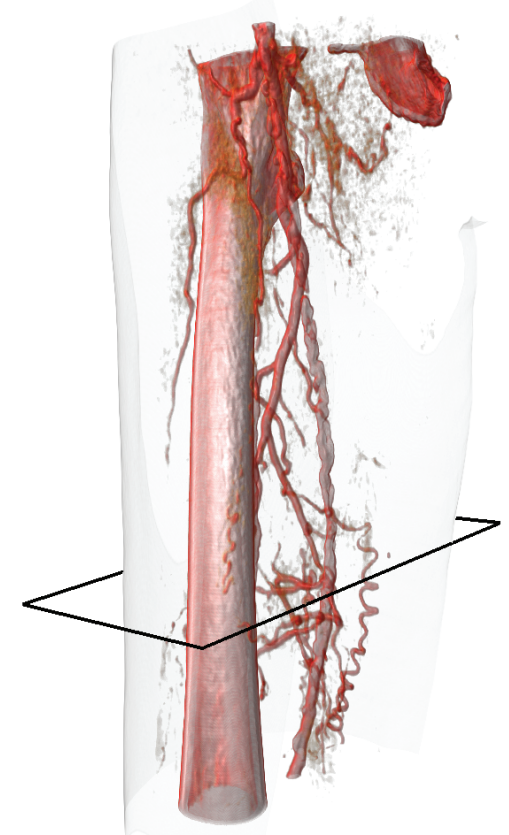

(d) Manually corrected optimization

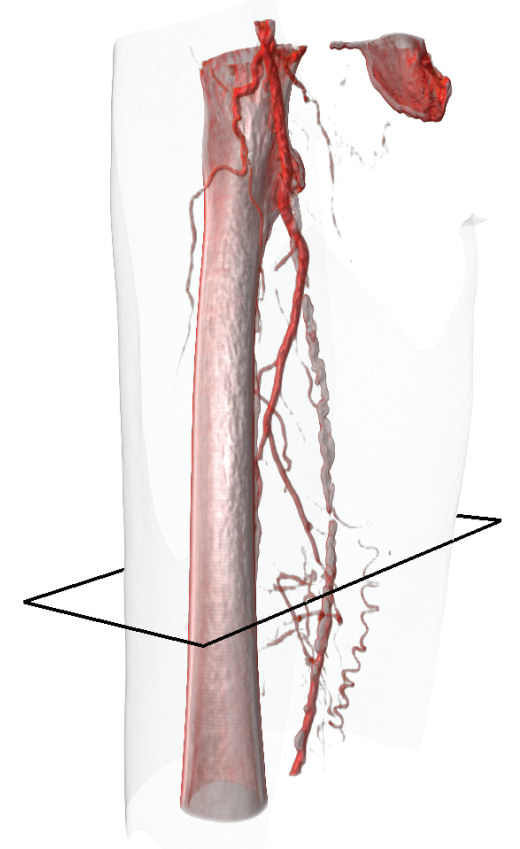

(b) Locally shifted TF preset

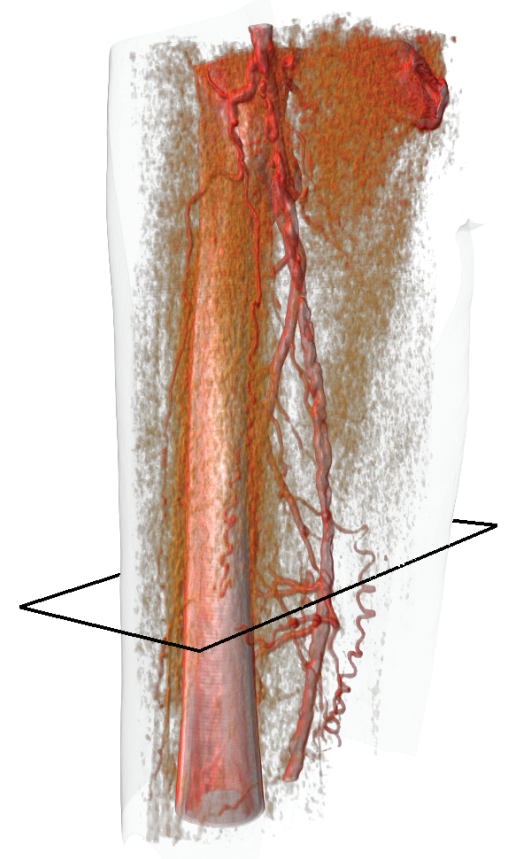

(c) Globally shifted TF preset

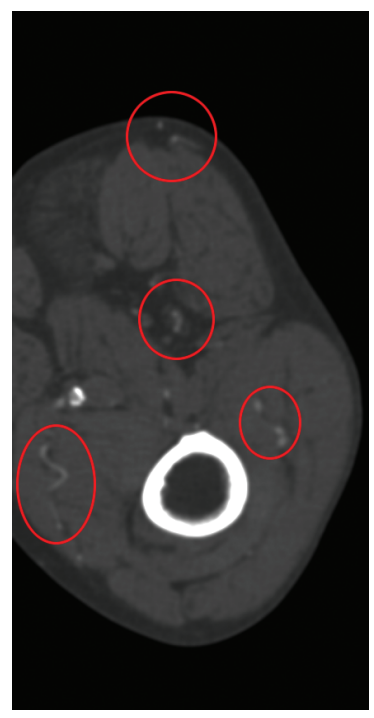

(e) Slice data

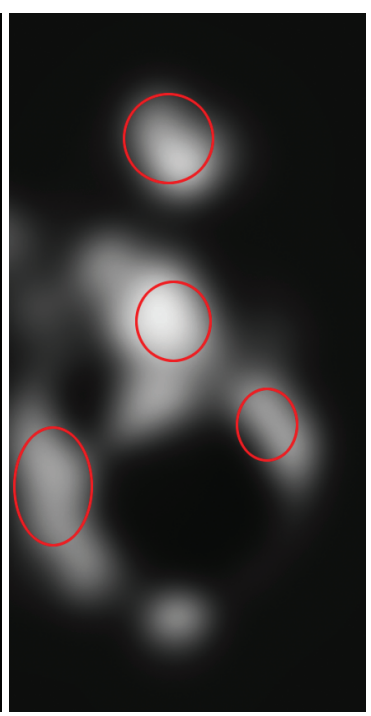

(f) Optimized parameters

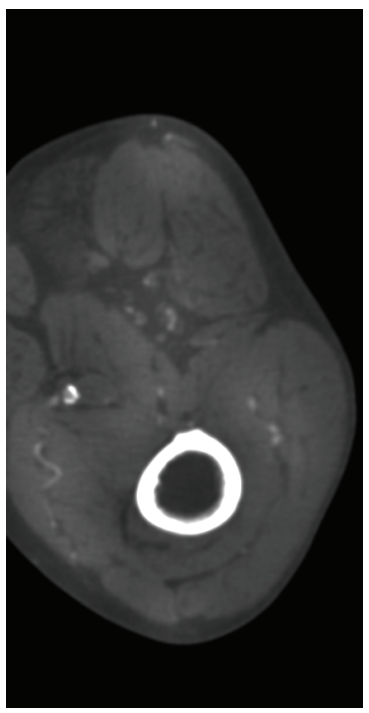

(g) Slice data shifted

Fig. 9. CTA of calcified vessels in leg. The contrast agent fails to reach the smaller vessels resulting in lower intensities in these structures. Because of this, the TF preset visualization misses these vessels in (a). Locally shifting the TF preset by our method reveals many of these vessels as shown in (b). This can be compared with (c) which is the result of globally shifting the TF manually. Note that (c) further reveals some weak intensity vessels not found in (b). If necessary, the user can manually correct (b) by globally shifting and scaling the optimized parameters, with the result in (d). This contains most of the vessels found in (c), with much reduced visual clutter. The slices (e-g) (indicated by the black frames in (a-d)) show how the TF preset is affected in (f). Correlating the vessels in the data slice (e) with the regions in the TF parameters (f) (for example the circled regions) gives examples of the localization of the optimization to the vessel structures. The slice (g) shows the shifts directly applied to the data, which is inversely equivalent to applying the shifts to the TF. 
Spatially close vessels with varying intensity: The outcome when two vessels with different intensities are spatially close together depends on the distance between them and the regularization. Compromises will be made if the distance is less than what is enforced by the regularization. In such cases, the parameters of the two vessels will be averaged, possibly giving an incompletely visualized vessel. We see an example of this in Figure 8(c), where the low intensity vein is partially visible in the upper left part of the optimized image. Typically, it is not important to study both non-contrast and contrast filled vessels simultaneously, but future user studies will verify if this is a practical problem for real applications.

Insufficiently suppressed background: There is, of course, no way to guarantee that the background is fully, or even sufficiently, suppressed in every single situation. This is further emphasized by the fact that the so called "optimal" TF really is a term dependent on the observer and his or her preferences. In the case that the background does obscure some important feature, the user has the possibility to perform a global shift of the optimized parameters, much like in Figure 9(d).

The loss of coherence between intensity and color: In the standard TF model, there is a strong connection between image intensity and output color. When the TF is allowed to change locally in the data, which it is in this work, this connection is lost. We discussed this issue with a few clinical professionals. The general agreement was that as long as they knew that the intensity-color connection was manipulated they deemed it as an acceptable trade-off for increased image quality. They did, however, stress that there should be a way to turn the feature off in case a situation arose where the true intensity was important.

\section{Summary AND Conclusions}

In this work we presented a method for automatically tuning TFs for visualizing contrast enhanced blood vessels. We illustrated the performance of the method using one synthetic and two real CTA datasets. These examples showed the method's capability of tuning a preset TF to match both spatially varying and generally shifted Hounsfield values of the data.

The idea is derived from observations on how physicians interact with the original image data to extract the clinically relevant information. It is based on a simple model with very few and physically motivated parameters. For these reasons we believe that the proposed method will be accepted in clinical routine.

In this study we controlled the value of the shift based on vesselness measures described in the literature. The approach described here is generic in the sense that the exact form of the criterion can be changed and other, similar, measures should be studied and optimized in future development of the method. An important part of the future work is also to validate the success of the method in controlled user studies.

\section{ACKNOWLEDGMENTS}

The presented concepts have been realized using the Voreen open source visualization framework (http://www. voreen.org).

\section{A Vesselness}

Denoting the eigenvalues of the Hessian as $\lambda_{1}, \lambda_{2}$ and $\lambda_{3}$, the vesselness by Sato assumes the ordering $\lambda_{1} \geq \lambda_{2} \geq \lambda_{3}$. These eigenvalues correspond to maximal and minimal second derivatives along the principal orientations of the structure in the data. A line structure in 3D can be characterized by three conditions: $\lambda_{3} \ll 0, \lambda_{3} \simeq \lambda_{2}$ and $\lambda_{2} \ll \lambda_{1} \simeq 0$. In [29], these conditions are expressed by:

$$
V_{\sigma}= \begin{cases}\left|\lambda_{3}\right|\left(\lambda_{2} / \lambda_{3}\right)^{\gamma}\left(1+\lambda_{1} /\left|\lambda_{2}\right|\right)^{\gamma} & \lambda_{2}<\lambda_{1} \leq 0 \\ \left|\lambda_{3}\right|\left(\lambda_{2} / \lambda_{3}\right)^{\gamma}\left(1-\alpha \lambda_{1} /\left|\lambda_{2}\right|\right)^{\gamma} & \left|\lambda_{2}\right| / \alpha>\lambda_{1}>0>\lambda_{2} \\ 0 & \text { otherwise }\end{cases}
$$

where $\sigma$ is the standard deviation of the Gaussian derivative kernels used to approximate the Hessian. The parameters $\gamma \geq 0$ and $\alpha \in(0,1]$ control the selectivity for different classes of local structure. In practice they can be used for dealing with deviations from the optimal conditions, e.g. when a vessel is stenotic. Typical values used for all examples in this text are $\gamma=1$ and $\alpha=0.25$.

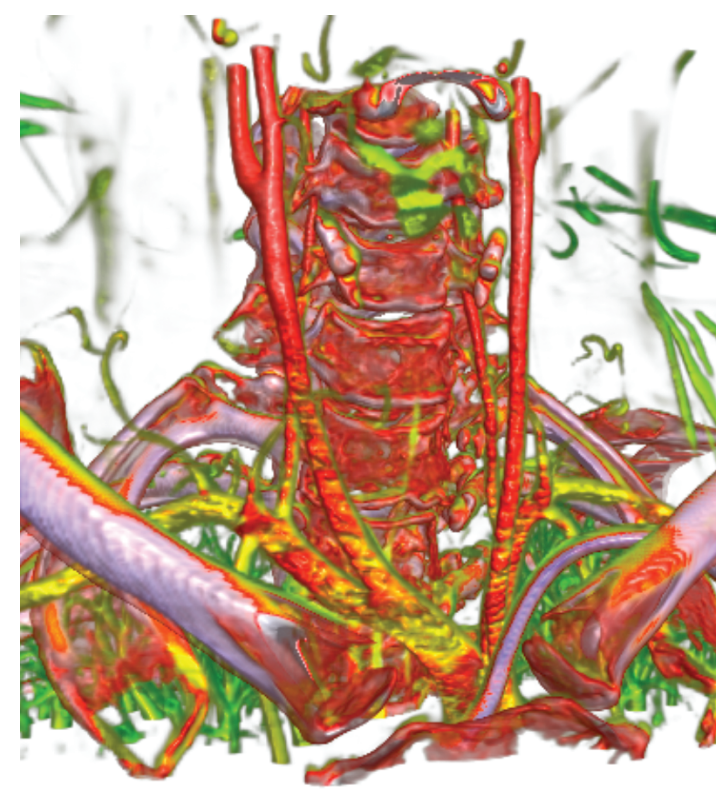

(a) Carotid dataset

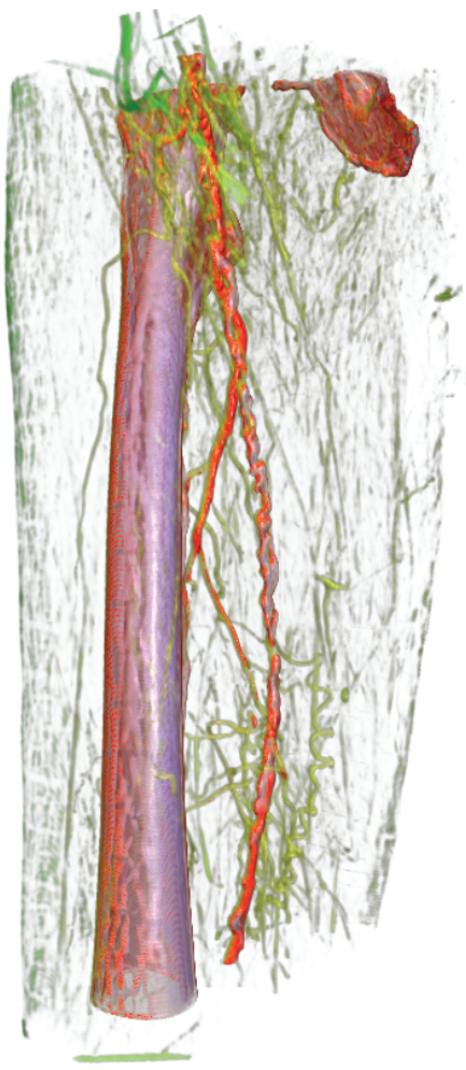

(b) Leg dataset

Fig. 10. Comparison renderings by the ideas presented by Joshi et al. [9]. This method uses the vesselness measure in the original data to "boost" the opacity of low intensity voxels. In these images, voxels which are below the TF, and therefore traditionally hidden in the visualization, are colored green-to-yellow. In (a) we see especially low intensity vessels in the lung appearing, and the lower parts of the carotid artery (refer to Figure 8). The leg in (b) can be compared to the results in Figure 9. 


\section{References}

[1] K. P. Andriole, R. L. Morin, R. L. Arenson, J. A. Carrino, B. J. Erickson, S. C. Horii, D. W. Piraino, B. I. Reiner, J. A. Seibert, and E. Siegel. Addressing the coming radiology crisis-the Society for Computer Applications in Radiology transforming the radiological interpretation process (TRIP) initiative. Journal of digital imaging the official journal of the Society for Computer Applications in Radiology, 17(4):235-243, 2004.

[2] K. Bühler, P. Felkel, and A. L. Cruz. Geometric methods for vessel visualization and quantification - a survey. In H. M. G. Brunnett, B. Hamann, editor, Geometric Modelling for Scientific Visualization. Springer Verlag, 2003.

[3] C. Correa and K.-L. Ma. Size-based transfer functions: A new volume exploration technique. Visualization and Computer Graphics, IEEE Transactions on, 14(6):1380 -1387, nov.-dec. 2008.

[4] R. A. Drebin, L. Carpenter, and P. Hanrahan. Volume rendering. In $S I G$ GRAPH '88: Proceedings of the 15th annual conference on Computer graphics and interactive techniques, pages 65-74, New York, NY, USA, 1988. ACM.

[5] K. Drechsler and C. Laura. Comparison of vesselness functions for multiscale analysis of the liver vasculature. In Information Technology and Applications in Biomedicine (ITAB), 2010 10th IEEE International Conference on, pages $1-5$, nov. 2010

[6] M. Haidacher, D. Patel, S. Bruckner, A. Kanitsar, and M. Groller. Volume visualization based on statistical transfer-function spaces. In Pacific Visualization Symposium (PacificVis), 2010 IEEE, pages 17 -24, mar. 2010.

[7] T. He, L. Hong, A. Kaufman, and H. Pfister. Generation of transfer functions with stochastic search techniques. In Proceedings of the 7th conference on Visualization '96, VIS '96, pages 227-ff., Los Alamitos, CA, USA, 1996. IEEE Computer Society Press.

[8] J. Hladuvka, A. König, and E. Gröller. Curvature-based transfer functions for direct volume rendering. In In Bianca Falcidieno, editor, Spring Conference on Computer Graphics 2000, pages 58-65, 2000.

[9] A. Joshi, X. Qian, D. Dione, K. Bulsara, C. Breuer, A. Sinusas, and X. Papademetris. Effective visualization of complex vascular structures using a non-parametric vessel detection method. IEEE Transactions on Visualization and Computer Graphics, 14:1603-1610, 2008.

[10] G. Kindlmann and J. W. Durkin. Semi-automatic generation of transfer functions for direct volume rendering. In Proceedings of the 1998 IEEE symposium on Volume visualization, VVS '98, pages 79-86, New York, NY, USA, 1998. ACM.

[11] G. Kindlmann, R. Whitaker, T. Tasdizen, and T. Moller. Curvature-based transfer functions for direct volume rendering: Methods and applications. In Proceedings of the 14th IEEE Visualization 2003 (VIS'03), VIS '03, pages 67-, Washington, DC, USA, 2003. IEEE Computer Society.

[12] C. Kirbas and F. Quek. A review of vessel extraction techniques and algorithms. ACM Comput. Surv., 36(2):81-121, 2004.

[13] J. Kniss, G. Kindlmann, and C. Hansen. Multidimensional transfer functions for interactive volume rendering. IEEE Transactions on Visualization and Computer Graphics, 8(3):270-285, 2002.

[14] C. Kubisch, S. Glaer, M. Neugebauer, and B. Preim. Vessel visualization with volume rendering. In L. Linsen, H. Hagen, B. Hamann, and H.-C. Hege, editors, Visualization in Medicine and Life Sciences II, Mathematics and Visualization, pages 109-134. Springer Berlin Heidelberg, 2012.

[15] D. Laidlaw, K. Fleischer, and A. Barr. Partial-volume bayesian classification of material mixtures in $\mathrm{mr}$ volume data using voxel histograms. Medical Imaging, IEEE Transactions on, 17(1):74 -86, feb. 1998.

[16] D. Lesage, E. D. Angelini, I. Bloch, and G. Funka-Lea. A review of 3d vessel lumen segmentation techniques: Models, features and extraction schemes. Medical Image Analysis, 13(6):819 - 845, 2009.

[17] M. Levoy. Volume rendering: Display of surfaces from volume data. Computer Graphics and Applications, IEEE, 8(3):29 -37, may. 1988.

[18] S. Lindholm, P. Ljung, C. Lundström, A. Persson, and A. Ynnerman. Spatial conditioning of transfer functions using local material distributions. Visualization and Computer Graphics, IEEE Transactions on, 16(6):1301 -1310 , nov.-dec. 2010.

[19] C. Lundström, P. Ljung, and A. Ynnerman. Local histograms for design of transfer functions in direct volume rendering. Visualization and Computer Graphics, IEEE Transactions on, 12(6):1570 -1579, 2006.

[20] C. Lundström and A. Persson. Characterizing visual analytics in diagnostic imaging. International Workshop on Visual Analytics, 2011.

[21] C. Lundström, A. Ynnerman, P. Ljung, A. Persson, and H. Knutsson. The alpha-histogram: Using spatial coherence to enhance histograms and transfer function design. In Proceedings Eurographics/IEEE Symposium on Visualization 2006, Lisbon, Portugal, pages 227-234, 2006.

[22] J. Marks, B. Andalman, P. A. Beardsley, W. Freeman, S. Gibson, J. Hodgins, T. Kang, B. Mirtich, H. Pfister, W. Ruml, K. Ryall, J. Seims, and S. Shieber. Design galleries: a general approach to setting parameters for computer graphics and animation. In Proceedings of the 24th annual conference on Computer graphics and interactive techniques, SIGGRAPH '97, pages 389-400, New York, NY, USA, 1997. ACM Press/AddisonWesley Publishing Co.

[23] D. Patel, M. Haidacher, J.-P. Balabanian, and E. Groller. Moment curves. In Visualization Symposium, 2009. PacificVis '09. IEEE Pacific, pages $201-208$, apr. 2009.

[24] H. Pfister, B. Lorensen, C. Bajaj, G. Kindlmann, W. Schroeder, L. Avila, K. Raghu, R. Machiraju, and J. Lee. The transfer function bake-off. Computer Graphics and Applications, IEEE, 21(3):16 -22, may/jun 2001.

[25] J.-S. Praßni, T. Ropinski, J. Mensmann, and K. H. Hinrichs. Shape-based transfer functions for volume visualization. In IEEE Pacific Visualization Symposium (PacificVis 2010), pages 9-16, mar 2010.

[26] B. Reiner. One size (doesn't) fit all. Journal of the American College of Radiology, 5(4):567 - 570, 2008

[27] S. Roettger, M. Bauer, and M. Stamminger. Spatialized transfer functions. In Proc. EuroVis '05, pages 271-278. IEEE Computer Society Press, 2005.

[28] T. Ropinski, J.-S. Praßni, F. Steinicke, and K. Hinrichs. Stroke-based transfer function design. In IEEE/EG Volume and Point-Based Graphics, pages 41-48, 2008.

[29] Y. Sato, C. Westin, A. Bhalerao, S. Nakajima, N. Shiraga, S. Tamura, and R. Kikinis. Tissue classification based on $3 \mathrm{~d}$ local intensity structures for volume rendering. Visualization and Computer Graphics, IEEE Transactions on, 6(2):160 -180, apr. 2000.

[30] S. Takahashi, Y. Takeshima, and I. Fujishiro. Topological volume skeletonization and its application to transfer function design. Graph. Models, 66(1):24-49, 2004.

[31] S. Tenginakai, J. Lee, and R. Machiraju. Salient iso-surface detection with model-independent statistical signatures. In Visualization, 2001. VIS '01. Proceedings, pages $231-238$, oct. 2001

[32] G. Weber, S. Dillard, H. Carr, V. Pascucci, and B. Hamann. Topologycontrolled volume rendering. Visualization and Computer Graphics, IEEE Transactions on, 13(2):330 -341, march-april 2007.

[33] Y. Wu and H. Qu. Interactive transfer function design based on editing direct volume rendered images. Visualization and Computer Graphics, IEEE Transactions on, 13(5):1027 -1040, sept.-oct. 2007.

[34] J. Zhou and M. Takatsuka. Automatic transfer function generation using contour tree controlled residue flow model and color harmonics. $\mathrm{Vi}$ sualization and Computer Graphics, IEEE Transactions on, 15(6):1481 -1488 , nov.-dec. 2009. 